\title{
Mgr Eugène Lapointe, initiateur du syndicalisme catholique en Amérique du Nord
}

\section{Jean-Claude Drolet}

Volume 33, 1966

URI : https://id.erudit.org/iderudit/1007321ar

DOI : https://doi.org/10.7202/1007321ar

Aller au sommaire du numéro

Éditeur(s)

Les Éditions Historia Ecclesiæ Catholicæ Canadensis Inc.

ISSN

0318-6172 (imprimé)

1927-7067 (numérique)

Découvrir la revue

Citer cet article

Drolet, J.-C. (1966). Mgr Eugène Lapointe, initiateur du syndicalisme catholique en Amérique du Nord. Sessions d'étude - Société canadienne d'histoire de l'Église catholique, 33, 47-56. https://doi.org/10.7202/1007321ar

Tous droits réservés @ Les Éditions Historia Ecclesiæ Catholicæ Canadensis Inc., 1967
Ce document est protégé par la loi sur le droit d'auteur. L'utilisation des services d'Érudit (y compris la reproduction) est assujettie à sa politique d'utilisation que vous pouvez consulter en ligne.

https://apropos.erudit.org/fr/usagers/politique-dutilisation/ 


\section{Mgr Eugène Lapointe, initiateur du syndicalisme catholique en Amérique du Nord}

Le début du XXe siècle marque au Canada français un effort d'action sociale admirable mais hélas trop peu connue. Des premières initiatives apparaissent: la première Caisse populaire à Lévis, l'Association catholique de la Jeunesse canadienne-française, l'Action Sociale Catholique, pour n'en citer que quelques-unes. Les Catholiques sociaux de la France, de l'Allemagne, de la Belgique, de l'Autriche, de l'Angleterre, de l'Italie et de la Suisse avaient affirmé et répété au XIXe siècle que la question sociale était morale et religieuse. Les Catholiques sociaux du Canada français, prêtres et laïcs, se sont empressés d'appliquer ce principe au milieu canadien-français. Ainsi le religieux, le national et le social ne font désormais qu'un. Des personnalités religieuses et laïques éveillent les Canadiens français à l'importance de l'économique et du social; des horizons nouveaux se dévoilent pour l'action collective. Les vingt premières années du XXe siècle constituent donc une période extraordinairement féconde pour le mouvement social catholique; cette période scelle l'union du religieux, du national et du social. C'est une fécondité qui répond d'ailleurs à Rerum Novarum de Léon XIII. Car ce document force les esprits sérieux à reconsidérer les problèmes du milieu canadien-français d'une façon nouvelle et plus adaptée. Parmi les personnalités religieuses et laïques, parmi les pionniers du mouvement social catholique au Canada se dégage la figure de Mgr Eugène Lapointe. Ce prélat du diocèse de Chicoutimi a joué en notre milieu le rôle des premiers chrétiens sociaux des pays d'Europe: l'évêque Ketteler, en Allemagne; le baron de Vogelsang, en Autriche; le comte de Mun, en France; le cardinal Manning, en Angleterre; le professeur Decurtins, en Suisse; le cardinal Gibbons, aux Etats-Unis. Qu'a-t-il réalisé cet humble prélat d'un diocèse du nord de la Province de Québec pour figurer dans la galerie des pionniers du mouvement social catholique au Canada français? C'est lui, Mgr Eugène Lapointe, qui a inauguré l'organisation syndicale catholique et nationale dans la Province de Québec et même dans toute l'Amérique du Nord.

Il est nécessaire de retracer brièvement la carrière de ce prêtre, éducateur, orateur, journaliste, apôtre social. Ce dernier aspect de sa personnalité, c'est-à-dire l'apôtre social, constituera toutefois la partie principale de cette étude.

Notes biographiques.

François-Eugène Lapointe est né à la Malbaie, comté de Charlevoix, le 21 avril 1860. « Par son origine, il tient donc à la terre qui 
produit le bon blé et les fortes générations; au pays de Charlevoix qui porte l'empreinte d'un Créateur puissant et audacieux, à l'un de ces foyers où les pensées de l'éternité préoccupent plus que celles du temps ${ }^{1}{ }^{\text {* }}$. D'ailleurs "il gardera toute sa vie le culte de ce pays, du terroir particulier où s'écoula son enfance paisible ${ }^{2}$ ». Jusqu'en 1874, le jeune Eugène Lapointe étudie à l'école du rang et en septembre de la même année, il entre au Petit Séminaire de Québec pour y faire ses études classiques. Pendant ces années, il se révèle un élève bien doué, travailleur et sérieux, aimant peu le jeu, préférant surtout la promenade avec des confrères plus agés que lui, entre autres avec un nommé Paul-Eugène Roy, futur archevêque de Québec. Son cours est particulièrement brillant, il devient secrétaire, puis président de l'Académie Saint-Denys. A sa sortie du Séminaire de Québec, Eugène Lapointe possède de bonnes connaissances générales, une culture littéraire vraiment remarquable et surtout un talent oratoire très prometteur. Le temps est enfin venu pour lui, comme pour ses confrères, de choisir sa carrière. L'étude du Droit semble l'intéresser, il caresse même l'ambition de devenir un jour premier ministre de la Province de Québec. Les autorités du Séminaire de Québec songent à l'envoyer étudier les lettres à Paris, à la suite de l'abbé Paul-Eugène Roy, son ami. Disant adieu à ces projets, à ces rêves de jeunesse, il entre au Grand Séminaire en septembre 1882. Mais, par sa naissance, il appartient au jeune diocèse de Chicoutimi, et le premier évêque, Mgr Dominique Racine, le réclame pour son séminaire. Car dans les profondeurs du Royaume du Saguenay se trouve o un séminaire jeune d'âge puisque fondé en 1873 - un séminaire riche d'espérances, mais pauvre de ressources matérielles, plus pauvres encore de personnel ${ }^{3}$ ». Comme c'est la coutume à l'époque, tout en étudiant la théologie, le jeune séminariste reçoit une carte de route des plus chargées. Tour à tour, durant ses années de cléricature, il enseigne les mathématiques de 1882 à 1883 , la philosophie de 1882 à 1886 , il exerce la fonction de surveillant de 1883 à 1886 . Le ler août 1886, c'est la réalisation de son idéal, il reçoit l'ordination sacerdotale des mains de Mgr Dominique Racine, dans l'église de la Baie Saint-Paul. Puis en septembre 1886, on le nomme professeur de philosophie au Petit Séminaire de Chicoutimi. Très tôt, ce jeune prêtre offrant des qualités remarquables, on lui confie les charges les plus importantes de Directeur des élèves et de Préfet des études, charges qu'il exercera jusqu'en 1891, année où il ira poursuivre des études en philosophie à Rome, au Collège Romain. Il en reviendra en 1893, avec un doctorat en philosophie, brillamment décroché.

Ces deux années d'études à Rome de $M$. l'abbé Lapointe nous intéressent au plus haut point, car c'est vraiment là, sans nul doute, qu'il sentira germer en lui cette vocation plus particulière d'apôtre social.

1 Mgr 0. D. Simard, Mgr Eugène Lapointe, Sa carrière dans Alma Mater, I (mai-juin 1936), p. 68.

2 R. Desgagné, ptre, Mgr Eugène Lapointe, Eloquence Religieuse, dans Saguenayensia, IV, 6 (1962), p. 135.

3 Mgr O. D. SIMARD, op. cit., p. 68. 


\section{Années de préparation.}

A l'automne 1891, M. l'abbé Lapointe arrive donc à Rome pour étudier la philosophie au Collège Romain sous la direction du célèbre Père Di Maria. Un jeune prêtre étudiant de Montréal, M. l'abbé Elie Auclair, qui sera le "compagnon de messe " de l'abbé Lapointe, nous le décrit ainsi:

Svelte, et de haute taille - qu'il n'avait guère tendance à courber dégagé d'allure, de démarche même un peu brusque, et un brin solennel, de figure au teint pâle et de traits réguliers, avec des yeux au regard pénétrant, un large front de penseur et des cheveux tout noirs, très fournis, M. Lapointe paraissait distant de prime abord. Mais, ce n'était là qu'une distinction de la dignité qu'il savait mettre en tout, et, sitôt qu'on l'avait pénétré, on le sentait si bon, si affable et si prévenant, qu'il n'y avait pas moyen de ne pas l'aimer aussi fortement que respectueusement. Et puis, quel charmant causeur il était aux heures de détente et de récréation! Les confrères le recherchaient volontiers. Jouir de son intimité était considéré par tous comme un privilège que, je m'en flatte, j'ai pu goûter largement ${ }^{4}$.

Travailleur consciencieux et méthodique, M. Lapointe profite heureusement des cours chez les Pères Jésuites du Collège Romain. « Il se montre - au dire de son confrère et ami, M. Auclair - un fidèle observateur du règlement de la maison d'étude qu'est le Collège Canadien. Il donne à tous l'exemple d'une parfaite régularité, sans ostentation

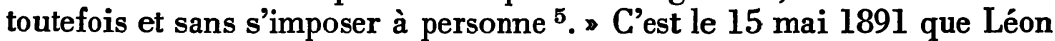
XIII publie son encyclique Rerum Novarum, si justement appelée la charte des travailleurs chrétiens. Sous la direction de l'éminent philosophe et sociologue qu'est le Père Di Maria, l'abbé Lapointe s'enthousiasme et s'imprègne de la doctrine sociale du grand Léon XIII doctrine si heureusement adaptée aux conditions modernes. Dès son départ pour Rome, l'abbé Lapointe est déjà sensibilisé aux problèmes sociaux de sa région et de sa province. D'ailleurs "sa vocation sociale naquit de bonne heure, alors que jeune écolier, puis étudiant au Séminaire de Québec, il fut témoin de scènes brutales, découlant de la misère ouvrière, et qu'il vit le sang couler. C'était aux alentours des années 1870-1875. Plusieurs conflits s'étaient produits dans les manufactures québecoises ${ }^{6}$. "Nous lisons dans ses Mémoires :

En acceptant d'aller suivre des cours à Rome, je m'étais bien proposé de ne pas me borner à des études de philosophie et de théologie. Mon âge - j'avais plus de trente ans - plusieurs années d'expérience dans la direction et l'éducation de la jeunesse, mes relations au Canada avec des personnes de tout rang déjà, m'avaient ouvert les yeux sur les problèmes sociaux qui se posaient dès lors. L'encyclique Rerum Novarum qui venait de paraître, m'avait vivement frappé. Je voyais déjà clairement qu'à des besoins nouveaux il fallait pourvoir par des institutions et des méthodes nouvelles ?.

4 Elie-J. AuclaIR, Lettre à M. le chanoine Edmond Duchesne, supérieur, dans Alma Mater, série III, 1-7 (mars 1936), p. 53.

5 Loc. cit.

6 Michel TÊtu, La Fédération Ouvrière Mutuelle du Nord, dans Relations Industrielles, octobre 1962, p. 403.

7 A.S.H.S. - Document 813, Mgr Eugène Lapointe, Mémoires, p. 240. 
Dans ses conversations avec ses confrères d'alors, les abbés Elie Auclair, Aristide Magnan et Alfred Lortie, M. Lapointe revient souvent sur la question sociale, c'est l'un de ses sujets de conversation favoris. Déjà il parle longuement de ses projets de réforme sociale en son pays du Saguenay et dans tout le Canada. M. Lapointe ne se contente pas de cours théoriques sur la question sociale et sur la condition des ouvriers, mais il veut surtout observer les expériences déjà commencées dans quelques pays d'Europe à la fin du XIXe siècle.

Co travail śtait commoneć on Europe, ćcrit il, dans ses Mómoircs. Je l'avais suivi de loin. Je voulais le voir sur place et en constater les résultats. C'était une étude d'observation que j'avais commencée à Rome et que je poursuivais en France durant mes vacances ${ }^{8}$.

Quelquefois seul, mais la plupart du temps, accompagné de ses amis, il parcourt l'Italie, se rend en Bretagne, séjourne à Lyon, à Bourges, à Tours, à Nantes, "moins soucieux de s'arrêter devant les monuments que de converser le plus possible avec les gens, en particulier avec ceux, prêtres ou laïques, dont les entretiens peuvent lui apporter quelque lumière sur les sujets d'ordre social, religieux et économique qui l'intéressent ${ }^{9}$ ».

Ce fut l'occasion d'observation du plus grand intérêt. Nous vivions la vie de France. Que de renseignements précieux nous puisâmes dans ces conversations presque journalières avec des hommes d'Eglise de tout rang, sans compter ce que nous révélaient de fréquents contacts occasionnels avec les laïques, surtout les paysans 10 .

Il est intéressant de noter que pour satisfaire pleinement sa curiosité, pour mieux observer, l'abbé Lapointe revêt un costume laïc; il lui est ainsi plus facile d'approcher les ouvriers, les paysans et de discuter de tous leurs problèmes. Au cours de tous ces voyages d'observation et certes de formation, l'abbé Eugène Lapointe pense à son pays. Souvent il cause avec les abbés Auclair et Lortie ades conditions pleines de dangers que créaient dans le pays, une presse neutre et un mouvement ouvrier neutre ${ }^{11} \bowtie$.

A son départ de Rome pour le Canada, la vocation sociale de l'abbé Lapointe est bien mûrie et édifiée sur des bases doctrinales solides. Il est préparé pour une féconde carrière. A partir de 1893, c'est un bilan assez compliqué que celui des activités de l'abbé Lapointe. Il enseignera les mathématiques un an, la philosophie quinze ans, la sociologie un an, la théologie pastorale vingt-neuf ans. Il sera directeur des élèves onze ans, préfet des études huit ans, procureur neuf ans, directeur spirituel trois ans, supérieur dix-huit ans. Sur le plan diocésain, il occupera aussi des postes de premier plan. Il sera vingt ans Vicaire général, soit du 18 septembre 1908 à 1928, presque un an Vicaire capitulaire; protonotaire apostolique en 1914; doyen du chapître de Chicoutimi en 1926. Le 19 mars 1912, il est nommé par Mgr

8 Loc. cit.

9 A.S.H.S., Mémoires de Mgr Lapointe, p. 240.

10 Ibid., p. 232-234.

11 Ibid., p. 232. 
Labrecque, Directeur général des Oeuvres sociales diocésaines; en septembre 1931, à la demande de Mgr Lamarche, il devient le premier Directeur diocésain de l'Action Catholique, charge qu'il occupera jusqu'en 1935.

Mgr Eugène Lapointe a été un éducateur doublé d'un homme d'action et de gouvernement. Le premier chapitre de ses activités nombreuses est celui de son apostolat social.

\section{Le Fondateur de la Fédération Ouvrière Mutuelle du Nord.}

Sur le plan social, Mgr Eugène Lapointe est un novateur à la fois prudent et hardi. Il sait regarder d'en haut et, par conséquent, voir loin, plus loin que la plupart de ses contemporains. Dès son arrivée d'Europe, il prend de nombreuses initiatives. Son premier et plus durable titre de gloire est d'être le fondateur de la première grande organisation ouvrière à base confessionnelle au Canada; il est le premier prêtre du Nouveau Monde à voir dans l'encyclique de l'immortel pontife autre chose qu'un magistral document. Il veut en appliquer la lumineuse doctrine.

Je fus témoin pour ma part, dit-il, d'inoubliables ovations au « Pape des ouvriers " au lendemain de la publication de cet immortel document. Avec quelle émotion j'ai vu chaque fois Léon XIII se pencher sur ces foules de travailleurs, les bras tendus comme pour les embrasser tous et les presser sur son cœur! Quelle lumière alors et quelle expression de tendresse dans son regard de feu 12 !

C'est donc vers 1903 que commence à s'élaborer le mouvement syndical catholique. Mais auparavant il faut mentionner ici le travail préparatoire d'éducation des ouvriers dirigé par Mgr Lapointe en vue des réalisations futures, travail consistant à former ces ouvriers aux idées d'union et de collaboration, à les entraîner aux responsabilités par l'établissement de caisses d'économie et de cercles d'études.

\section{Convaincu de la vérité de ces paroles de Léon XIII :}

Dans cet état de chose, les ouvriers chrétiens n'ont plus qu'à choisir entre ces deux partis: ou de donner leur nom à des sociétés dont la religion a tout à craindre, ou de s'organiser eux-mêmes et de joindre leurs forces pour pouvoir secouer hardiment un joug si injuste et si intolérable 13 .

Il décide d'organiser les travailleurs canadiens d'après les directives pontificales. EEuvre hardie, qui demande une volonté énergique, un homme de caractère et aussi de commandement.

Mon plus grand ennui, écrira-t.il au député Joseph Girard, à Ottawa, vient de ce que j'ai trop à faire par ailleurs et que je serai à peu près seul pour commencer cette oeurre. Mais, c'est égal, une fois pris, j'irai au bout ${ }^{14}$.

12 A.S.H.S. - Document 760-G, Mgr Eugène LAPorNTE, Le travail chrétien à Rome, p. 2.

13 Lettre encyclique de Léon XIII sur la condition des ouvriers, p. 40, Editions Apostolat de la Presse, Sherbrooke.

14 A.S.H.S. - Document 359, Lettre de M. Eugène Lapointe au député Jos Girard, à Ottawa, en date du 18 avril 1907. 


\section{C'est un vrai syndicat que veut fonder l'abbé Eugène Lapointe.}

Le devoir des ouvriers catholiques est donc tout tracé : ils doivent, s'ils veulent voir trompher leurs justes réclamations, grouper leurs forces dans des associations catholiques. Ce devoir s'impose surtout aux catholiques du Canada 15.

S'adressant aux ouvriers du Congrès des Travailleurs catholiques le 12 août 1903, il leur dit :

C'est de cette soif de la justice et de la charité que sont nés vos

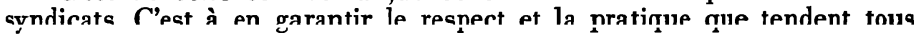
leurs efforts. C'est une pensée de justice et de charité qui vous réunit en Congrès et qui, $j$ 'en suis sûr, inspirera vos délibérations et dictera vos résolutions. Laissez-moi vous féliciter. Vous êtes dans la vraie voie. Et si le monde du travail peut être réformé selon la vérité, la justice et la charité pour son plus grand bonheur et pour la paix sociale, il le sera par vous et par ceux qui vous ressemblent 16 .

L'industrie naissante au Saguenay excite déjà la convoitise de la grande union neutre américaine, appelée l'Internationale. Mgr Lapointe fonde une véritable association ouvrière catholique " parce que, écrit-il, nos " associations étrangères" reposent toutes sur de faux principes non seulement religieux et moraux mais encore « sociaux et économiques * et que le contrôle de l'autorité religieuse dans de telles associations est nul et ne peut être que nul. Les principes qui régissent l'association à tous les degrés et dans toutes les sphères, se puisent à deux sources : le christianisme et le rationalisme. Il y a deux sciences économiques : l'une, la vraie, que l'on puise dans l'enseignement de l'Eglise et les ouvrages des sociologues chrétiens: l'autre, la fausse qui est le produit des cervaux en ébullition de la libre-pensée, et dont vous avez la quintessence dans tous les règlements des sociétés neutres dites internationales ${ }^{17}$. La correspondance de Mgr Eugène Lapointe, conservée aux archives de la Société historique du Saguenay, nous permet de croire que la préparation concrète de la Fédération Ouvrière du Chicoutimi débute vers le mois d'avril 1907. Dès novembre de la même année, le règlement de cette première association ouvrière catholique est prêt; ce n'est pas un travail absolument personnel, M. Lapointe a étudié longtemps les institutions similaires d'Europe et des Etats-Unis, mais il rédige ce règlement avec la collaboration de plusieurs citoyens de Chicoutimi, entre autres les avocats Onésime Tremblay, Simon Lapointe, F. X. Gosselin, le notaire Maltais, M. R. H. Beaulieu, l'ingénieur Taché et quelques autres.

J'ai essayé, écrit-il, de mettre dans ce règlement tout ce que l'expérience et l'étude ont pu m'apporter de lumière et d'utiles suggestions; quand vous l'aurez étudié, je crois que vous conviendrez qu'avec ce programme, des hommes de bonne volonté peuvent faire du bien à notre classe ouvrière ${ }^{18}$.

15 A.S.H.S. - Document 759-L, Sermon au Congrès des Travailleurs catholiques à Saint-Malo, Québec, le 12 août 1903 , II॰ partie, p. 7.

16 A.S.H.S. - Document 759-L, ibid., II' partie, p. 1-2-3.

17 A.S.H.S. - Document 359-G, Lettre de M. l'abbé E. Lapointe à M. Jos. Girard, à Ottawa, datée du 11 décembre 1907, p. 5.

${ }_{18}^{18}$ A.S.H.S. - Document 359-B, Lettre du même au même, du 25 novembre 1907. 
L'une des principales préoccupations de Mgr Lapointe est de soustraire cette union d'une part à l'influence des passions politiques et municipales, influence qui divise toujours, selon lui, et d'autre part à l'action des compagnies industrielles et des patrons. Le fondateur de cette oeuvre audacieuse est convaincu, à l'avance, de ne pas susciter la sympathie de tout le monde et surtout de ne pas être compris, même à Chicoutimi :

Je n'ai pas besoin de vous dire pourquoi, mais c'est égal; je poursuivrai mon cuvre en dépit des défections et des oppositions sourdes ou avouées. Je suis libre comme l'air; je ne dois rien à personne, je n'ai personnellement besoin de personne ${ }^{19}$.

Enfin le 22 décembre 1907 a lieu une première assemblée des membres inscrits sur la liste d'union. Les cinq membres du Conseil de Direction sont élus le 27 décembre, M. l'abbé Lapointe en est le secrétaire. L'oeuvre prend lentement racine et se poursuit au moins jusqu'en 1911. Nous lisons dans les Statuts et Règlements de la Fédération Ouvrière de Chicoutimi au chapitre premier à l'article deux ses objectifs :

Elle a pour objet l'étude, la protection et le développement des intérêts moraux et matériels de ses membres.

\section{Dans l'esprit de son fondateur et de ses collaborateurs, la Fédération} doit aussi :

s'occuper spécialement d'améliorer la situation économique des ouvriers dans les limites de sa circonscription, en encourageant, dans la mesure de ses moyens la mutualité catholique et nationale; en favorisant la création en dehors d'elle de sociétés coopératives de consommation et de crédit, d'une bourse du travail, qui garantira le travailleur honnête contre le chômage et assurera à l'employeur des ouvriers honnêtes et compétents.

la Fédération doit aussi favoriser la création :

d'une caisse d'économie populaire, d'unions professionnelles, d'écoles spéciales, où les jeunes gens qui ont fait leur cours modèle ou commercial dans les institutions déjà établies, acquerront, avant leur entrée en apprentissage, les connaissances techniques qui leur seront indispensables dans l'exercice de leur profession; en organisant, en un mot, le travail suivant les principes de la justice, de l'équité et de la charité, en conformité des lois du pays et en parfaite soumission aux directions de l'Eglise notamment à celles données par les Papes Léon XIII et Pie X 20 .

Evidemment le champ d'opération assigné à l'association est très vaste. L'Union ouvrière fondée par Mgr Lapointe est essentiellement catholique; elle entend professer la doctrine catholique intégrale et s'inspirer, dans son action et pour la solution des problèmes économiques et sociaux, de l'enseignement officiel de l'Eglise.

Dans le procès-verbal de la deuxième Assemblée Générale tenue le 19 janvier 1908, M. L'abbé Lapointe écrit :

Une œuvre de ce genre, d'un caractère tout nouveau, ne pouvait manquer de passer au creuset de l'épreuve. La Fédération eut son heure douloureuse. Un moment on put croire qu'elle allait sombrer 21 .

19 A.S.H.S. - Document 359-B, Lettre de M. Lapointe au député Girard, datée du 25 novembre 1907, p. 2.

20 A.S.H.S. - Document 259, Statuts et Règlements de la F.O.C., 1907, p. 1-2.

21 A.S.H.S. - Document 718, Procès-Verbal de F.O.N., 1914-1918, p. 19. 
Cette épreuve arrive dès 1911, la Fédération est à l'agonie et ne garde plus qu'une existence officielle. Hélas les espérances du fondateur de la Fédération de Chicoutimi ne sont pas réalisées. La cause première de cet échec vient de l'indifférence générale des travailleurs à l'égard de l'organisation ouvrière; les uns redoutent l'ingérence du clergé dans les questions du travail, l'amitié de M. Lapointe pour un grand industriel J. E. A. Dubuc leur semble suspecte; d'autres ouvriers ne veulent pas y entrer par crainte de déplaire à leurs employeurs et de perdre leur place; l'esprit de partisannerie politique contribue aussi à paralyser l'association ouvrière. Il faut signaler le travail de quelques dirigeants de l'Internationale et de certains individus ou groupes contre le clergé et les Canadiens français. Une autre cause de faiblesse vient de la constitution même de la Fédération qui veut imiter les expériences tentées en Europe surtout les cercles ouvriers établis par Albert de Mun où patrons et ouvriers se rencontrent pour discuter leurs problèmes; ce système n'est pas très apprécié par des ouvriers de Chicoutimi ${ }^{22}$. Le caractère de la fondation de Mgr Lapointe est enfin trop idéal, car - comment concevoir une union formée d'ouvriers qui par la seule collaboration de quelques employeurs ou hommes de loi dans le bureau de direction, va pouvoir faire office de " tribunal de conciliation ${ }^{23}$ ? \$ Devant toutes ces difficultés, Mgr Lapointe part pour l'Europe afin de faire des enquêtes en France, en Allemagne, en Belgique, sur les organisations ouvrières. Il en revient avec la formule du syndicat ouvrier. Le 18 mai 1912 la Fédération Ouvrière de Chicoutimi devient la Fédération Ouvrière Mutuelle du Nord. Le 21 décembre 1912 la corporation de la Fédération reçoit sa charte civile. C'est l'aurore d'une grande activité qui se poursuivra jusqu'en 1914. Pendant ces années M. l'abbé Lapointe passe ses journées et ses soirées avec les ouvriers; il publie de nombreux articles dans les journaux; il livre un dur combat contre des adversaires de son œuvre. Ainsi en 1914, il a vaincu son principal ennemi : le Congrès des Métiers et du Travail du Canada, \& ce vaste syndicat, écrit-il, de presque toutes nos sociétés ouvrières qui n'est au fond qu'une organisation politique et une monstrueuse exploitation 24 . C'est un travail immense de propagande et d'organisation accompli par Mgr Lapointe. A la fin de sa vie, il avouera :

Durant ma longue carrière sacerdotale, mon dévouement s'est exercé dans bien des milieux, mais dans aucun, je n'ai éprouvé autant de bonheur, en dépit, remarquez-le bien, d'insurmontables difficultés, souvent, de crucifiantes déceptions aussi, qu'au milieu des ouvriers, parce que là plus que n'importe où ailleurs, $j$ 'ai senti mon cœur battre à l'unisson avec le cœur du frère du Christ par excellence, le pauvre, le faible, le déshérité, le méconnu qu'a été longtemps le travailleur manuel 25 .

22 M. Ludovic MaltaIs, Les syndicats catholiques, p. 19.

23 Michel TÊTU, La Fédération Ouvrière Mutuelle du Nord, p. 413.

24 A.S.H.S. - Document 359-G, Lettre de M. Lapointe à M. Girard, du 11 décembre 1907, p. 7.

25 A.S.H.S. - Document 760-P, Discours de Mgr Lapointe le 5 septembre 1943, p. 3, par. 3. 
Mais cette œuvre semée dans les difficultés, les oppositions et les déceptions commence à produire des fruits partout ailleurs dans la province. Comme il le rappelle dans la dernière conférence qu'il prononce, le 5 septembre 1943, au local des Syndicats de la rue Morin à Chicoutimi, dès 1912 des syndicats s'implantent ailleurs, d'abord à Hull, puis à Trois-Rivières, à Sherbrooke, à Saint-Hyacinthe, etc. Enfin, en 1921, de ces groupements isolés et plus ou moins vivants naît la Confédération des Travailleurs catholiques du Canada ${ }^{26}$.

Mgr Lapointe aime les ouvriers et il en est aimé aussi. Se réjouissant des progrès accomplis pour le règlement de la classe ouvrière, il dit :

Je verrai enfin l'ouvrier, mon frère, vivre pleinement durant un jour sur sept, comme tout le monde, sa vie d'homme, de citoyen, de chrétien. $\mathrm{Je}$ le verrai le dimanche et les jours de fête, se reposer comme tout le monde, tranquille, dans la douceur de la vie de famille 27.

Pour reconnaître le mérite de ce prélat en 1943, à l'occasion du 40e anniversaire de la fondation des syndicats catholiques, l'Université de Montréal lui décerne un doctorat honorifique en Sciences sociales, économiques et politiques. Mgr Eugène Lapointe est un apôtre social non seulement par l'organisation ouvrière catholique mais aussi par ses luttes pour la tempérance et pour assurer le respect du dimanche. Il est en outre le promoteur des retraites fermées dans le diocèse de Chicoutimi; il fait partie, en 1920, de la Commission centrale des Semaines sociales et y prononce trois importantes conférences en 1920, 1921, 1922. Mgr Lapointe possède en plus, à un rare degré les qualités qui font de lui un bon journaliste et un orateur recherché.

Si l'action sociale, enfin, est fondée à Québec, je puis bien vous dire à vous, que j'ai ma modeste part dans ce travail de longue haleine qui l'a préparé et fait aboutir 28 .

La Fédération Ouvrière de Chicoutimi et par la suite la Fédération Ouvrière Mutuelle du Nord est la première expérience d'organisation ouvrière catholique tentée au Canada, et dans une région où l'unionisme est plus connu de nom que de fait. En résumé, la Fédération a démontré la possibilité de l'organisation ouvrière catholique, son efficacité économique et ses bienfaits sociaux, au moins dans un milieu approprié et une région à population homogène ${ }^{29}$.

\section{Conclusion}

Mgr Eugène Lapointe fut un pionnier, un défricheur sur un terrain particulièrement difficile. Il a livré un dur combat mais il était de cette race des forts capables de puiser dans leur dévouement, leur ténacité et leurs convictions solides toutes les énergies nécessaires pour atteindre un objectif, pour réaliser ses visions sociales. Nous lisons dans l'Action

26 A.S.H.S. - Document 760-P, Discours de Mgr Lapointe, le 5 septembre 1943 , p. 3, par. 3.

27 R. DEsGaGné, op. cit., p. 137.

28 A.S.H.S. - Document 358-G, Lettre au député Girard du 11 décembre 1907 , p. 11 .

29 L. Maltais, op. cit., p. 15-17. 
catholique du 28 mai 1936 cet article de Jules Dorion, intitulé • Un Canadien d'avant-garde $:$ :

Mgr Lapointe est de la lignée de ces pasteurs qui ne se bornent pas à exercer leur ministère sacerdotal, mais ils vont hardiment de l'avant... Il a été un prêtre modèle, un directeur d'âmes au doigté merveilleux, un professeur émérite, mais encore un de ces entraîneurs qui aiguillonnent les volontés et savent leur donner de la trempe ${ }^{30}$.

Mgr Eugène Lapointe a été vraiment un pionnier du mouvement social catholique au Canada français, un apôtre du syndicalisme catholique et de l'aciion caiholique, un luïeur infatigable, à l'aliure queiquefois guerrière, dans les assemblées ouvrières contradictoires; sa parole, sa doctrine, son action ont eu une influence puissante, dans sa patrie du Saguenay et dans la province de Québec. Sa vie a été féconde pour l'Eglise et la Société.

\author{
Jean-Claude Drolet \\ Centre d'Etudes et de \\ Recherches Historiques \\ du Saguenay, \\ Chicoutimi, P.Q.
}

30 A.S.H.S. - Dossier 352, Action catholique, $28 \mathrm{mai} 1936$. 